\title{
Platinum Based Material for Additive Technology of Gas Sensors +
}

\author{
Alexey A. Vasiliev 1,2,3,*, Vitaliy P. Kim ${ }^{4}$, Sergey V. Tkachev 4,5, Denis Yu. Kornilov ${ }^{4}$, \\ Sergey P. Gubin ${ }^{4,5}$, Ivan S. Vlasov ${ }^{2}$, Igor E. Jahatspanian ${ }^{3}$ and Alexy S. Sizov ${ }^{6}$ \\ 1 National Research Center “Kurchatov Institute”, Moscow 123182, Russia \\ 2 Moscow Institute of Physics and Technology, Dolgoprudny, Moscow region 141701, Russia; \\ isvlasov5@yandex.ru \\ 3 St. Petersburg ITMO University, International laboratory “Sensor Systems”, St. Petersburg 197101, Russia; \\ drjie@mail.ru \\ 4 LLC AkKo Lab, Moscow 129110, Russia; vp.kim@physics.msu.ru (V.P.K.); tkachev_svmsu@mail.ru (S.V.T.); \\ info@akkolab.com (D.Y.K.); gubin@igic.ras.ru (S.P.G.) \\ 5 Kurnakov Institute of General and Inorganic Chemistry of Russian Academy of Sciences, Moscow 119991, \\ Russia \\ 6 Enikalopov Institute of Synthetic Polymeric Materials of RAS, Moscow 117393, Russia; \\ sizov@printeltech.com \\ * Correspondence: A-A-Vasiliev@yandex.ru; Tel.: +7-903-194-95-51 \\ + Presented at the Eurosensors 2018 Conference, Graz, Austria, 9-12 September 2018.
}

Published: 20 December 2018

\begin{abstract}
We prepared platinum nanoparticle ink usable for the fabrication of MEMS microheaters of high-temperature gas sensors and thermoresistors operating up to $450{ }^{\circ} \mathrm{C}$ and present some preliminary results on the application of the ink in sensor microheater manufacturing. The ink consists of platinum particles (3-8 $\mathrm{nm}$ ) suspended in ethylene glycol solution of polyvinylpyrrolidone. The ink is usable in both InkJet and AerosolJet printers. The annealing at temperature of about $600{ }^{\circ} \mathrm{C}$ leads to the formation of uniform microheater structure. The experiments on microheater aging confirm the stability of the printed microstructure at $450{ }^{\circ} \mathrm{C}$ for at least one year of operation. The substrates used for printing were thin alumina and LTCC ceramics with thickness of 12-20 $\mu \mathrm{m}$.
\end{abstract}

Keywords: Pt based nanomaterial; printing additive technology; sensor microheater; ceramic MEMS

\section{Introduction}

The application of additive (printing) technologies for the fabrication of high temperature sensors is very attractive because of simplicity of the method enabling the fabrication of fine (down to $10 \mu \mathrm{m}$ size) elements of sensor on almost any type of substrate: ceramics, polymeric films, anodized metals, etc. However, the use of printing technologies applying inks made from $\mathrm{Ag}, \mathrm{Au}, \mathrm{Pt}$ nanoparticles for the fabrication of high-temperature MEMS metal oxide and thermocatalytic gas sensors, applicable for the detection of hardly oxidized gases like methane, is restricted by two main factors. The first one is poor adhesion of high-temperature, mainly platinum, ink to existing MEMS platforms based on $\mathrm{SiO}_{2} / \mathrm{Si}_{3} \mathrm{~N}_{4}$. This problem can be solved by the application of non-silicon substrates [1], for example thin alumina or LTCC (low temperature co-fired ceramics) for the fabrication of MEMS platforms. Another factor is related with unavailability of materials for high temperature inks of printed microheaters. Now the ink based on Pt nanoparticles is available mainly from Fraunhofer-IKTS (Dresden) [2], this single producer has a possibility to dictate the conditions to the customers. The main technical disadvantage of the ink [2] is very wide distribution 
of nanoparticle size ranging from few nanometers to $500 \mathrm{~nm}$. This leads to the problems with print stability because the annealing at temperature of $600{ }^{\circ} \mathrm{C}$ recommended by Fraunhofer IKTS is not sufficient to get stable resistance of microheater.

Another problem is the control of wettability of the surface of LTCC or alumina substrate with the ink. The modification of this commercial ink seems to be impossible because of politics of the company neglecting any direct contacts with customers, therefore the only opportunity for them is the development of new ink with improved formulation.

All these issues forced us to think about the development of our own Pt inks usable in InkJet and AerosolJet printers giving high quality for relatively low cost of printed sensing elements.

In the main part of recent researches, e.g., [3,4], the authors used polyimide film as a substrate for the sensors made using InkJet and AerosolJet printing. This polymeric material permits the application of gas sensor at rather high working temperature-up to $350{ }^{\circ} \mathrm{C}$-and enables the fabrication of flexible devices free of some disadvantages of silicon MEMS ones. However, the application of both gold and polyimide restrict the operation temperature of the microheater by temperature of approximately $350^{\circ} \mathrm{C}$.

The target of this work was the development of Aerosol and InkJet printable ink consisting of nanoparticles of platinum suspended in a vehicle with viscosity of $5-10 \mathrm{cP}$. This ink should be suitable for the fabrication of ceramic MEMS devices operating at least up to $450{ }^{\circ} \mathrm{C}$.

\section{Experiment}

Nanoparticle ink containing 10-20 wt.\% of 3-8 nm Ag, Au, and Pt organic vehicle were prepared by wet method and optimized for the application in Dimatix DMP 2831 printer and for AerosolJet printer AJ 15XE (Neotech-AMT/Optomec, Nuremberg, Germany).

These instruments are, probably, optimal for microheater fabrication because they can give prit width down to $10 \mu \mathrm{m}$. AerosolJet printer working principle is close to traditional aerograph technique. The printable ink is atomized by purified air flow to give airborne droplets $1-5 \mu \mathrm{m}$ in diameter. This air flow direct the flow of aerosol to the nozzle, where the flow is additionally focused by air flow to give jet diameter of about 10-20 $\mu \mathrm{m}$. The jet remains focused up to distance of about $10 \mathrm{~cm}$ enabling contactless printing over 3D substrates. The main advantages of ink and aerosol jet techniques are (1) the minimization of the consumption of precious metals compared to sputtering technique, (2) the use of maskless technology, and (3) the contactless printing process at the distance between printing head and the substrate of several millimeters not damaging 10-20 $\mu \mathrm{m}$ thick alumina membrane used for MEMS fabrication.

The ink contained individual nano-particles of platinum without agglomeration. To prepare this ink, platinum in diluted solution of $\mathrm{H}_{2} \mathrm{PtCl}_{6}$ was reduced by ethylene glycol in presence of PVP (polyvinylpyrrolidone) and concentrated then using centrifuge (8000 rpm). TEM and electron diffraction pictures of nano-particles of $\mathrm{Pt}$ with size of $3-8 \mathrm{~nm}$ is presented in Figure 1 . The inks viscosity is of about $10 \mathrm{cP}$.

The printed microheater was first dried at temperature of $100{ }^{\circ} \mathrm{C}$ and then sintered at $600{ }^{\circ} \mathrm{C}$ for $1 \mathrm{~h}$. This relatively low sintering temperature is sufficient for the formation of platinum line consisting of 5-8 $\mathrm{nm}$ particle of platinum.

The platinum microheaters were deposited onto thin alumina membrane obtained by anodic oxidation of aluminium; the free standing alumina film was annealed at $1000{ }^{\circ} \mathrm{C}$ for $10 \mathrm{~h}$ used for membrane stabilization, removal of surface contaminations and improvement of the surface wettability by the ink. Resulting semi-transparent alumina with thickness of $12 \mu \mathrm{m}$ is robust enough to be used as a substrate for both InkJet and AerosolJet printing. Another material used as a substrate was LTCC obtained from Slovenian company KEKO (Ljubljana, Slovenia). This material could be prospective because of possibility of low cost mass production of ceramic MEMS based on this ceramics. 

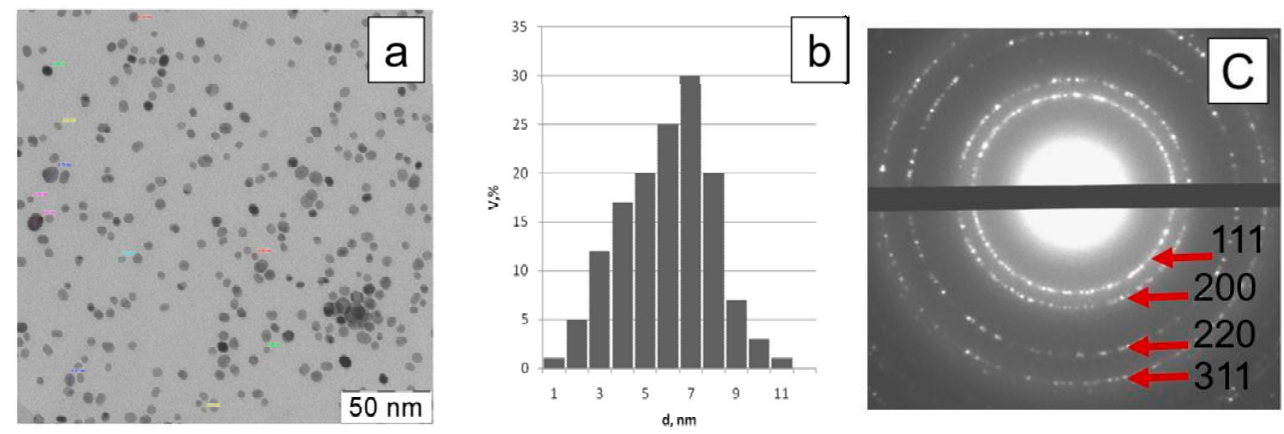

Figure 1. Nano-particles of platinum included in the ink for aerosol and ink-jet printing. (a) TEM image of platinum nano-particles, (b) size distribution histogram of the synthesized platinum nanoparticles, (c) diffraction pattern of electrons in the sample of platinum nano-particles synthesized in this work. Numbers indicate the numbers of the crystallographic planes of the platinum crystal phase referring to the corresponding reflections.

\section{Results and Discussion}

Pt film made of the ink using Aerosol and InkJet technique had thickness 1-2 $\mu \mathrm{m}$. It was studied after annealing at different temperatures. Figure 2 presents SEM images of the Pt coating on alumina ceramics after annealing at different temperatures. Increase in annealing temperature leads to an increase in crystallite size, but the coating remains unbroken after treatment.
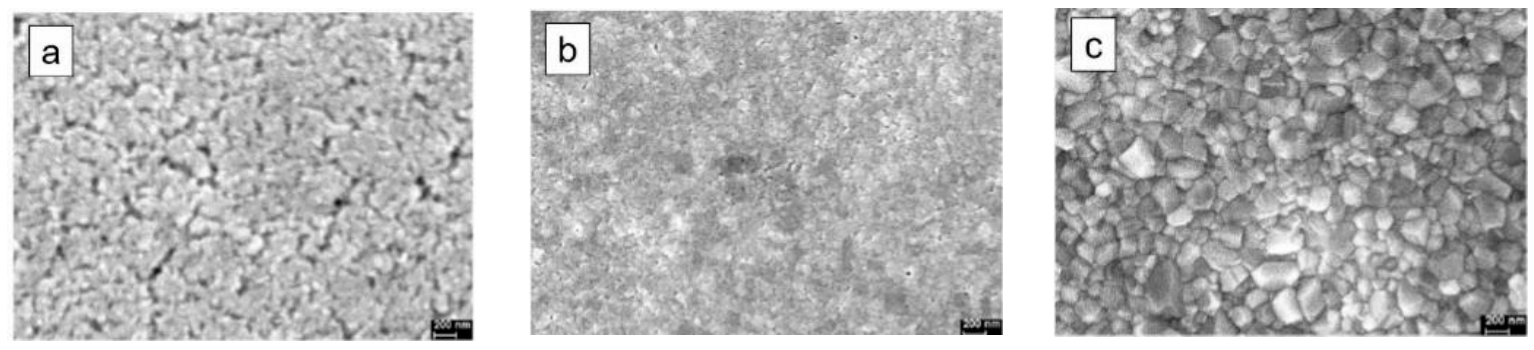

Figure 2. SEM-images of platinum nano-ink deposited onto alumina substrate after annealing in air: (a) thermal treatment at $200{ }^{\circ} \mathrm{C}$; (b) thermal treatment at $500{ }^{\circ} \mathrm{C}$; (c) thermal treatment at $800{ }^{\circ} \mathrm{C}$.

For the printing of narrow lines, Pt containing ink was inserted to the reservoir of ink jet printer Dimatix DMP 2831 and printed using a single nozzle of the instrument. The printing onto pre-annealed ceramic film gives high quality print with superficial resistance of about $2 \mathrm{Ohm} / \mathrm{square}$ for ceramic MEMS devices. Similar results were obtained using the Aerosoljet printing machine. After annealing at $350^{\circ} \mathrm{C}$, platinum film is characterized by sufficient adhesion to a glass substrate to pass "scotch test". For the application in gas sensor, a layer of platinum nano-particles is sintered about $600^{\circ} \mathrm{C}$.

The structure used for the resistivity and power consumption tests is presented in Figure 3A. This was a cantilever structure cut by 10 -Watt fiber optic laser from whole $12 \mu \mathrm{m}$ thick alumina substrate made by anodic oxidation of alumina. Resulting triangle shaped microheater was fixed in TO-8 packaging using $20 \mu \mathrm{m}$ platinum wires. Similar printing results were obtained using thin LTCC membrane as a substrate. In this case, thin membrane was fixed by glass over the hole in 0.5 mm thick LTCC substrate.

The width of the lines in the triangle apex part is of $40-50 \mu \mathrm{m}$, the microhotplate area is of $\sim 300$ $\times 300 \mu \mathrm{m}$, power consumption of $\sim 70 \mathrm{~mW}$ at $450{ }^{\circ} \mathrm{C}$ is comparable with those of silicon-technology based microheaters [5]. Thermal response time is of $\sim 50 \mathrm{~ms}$. 

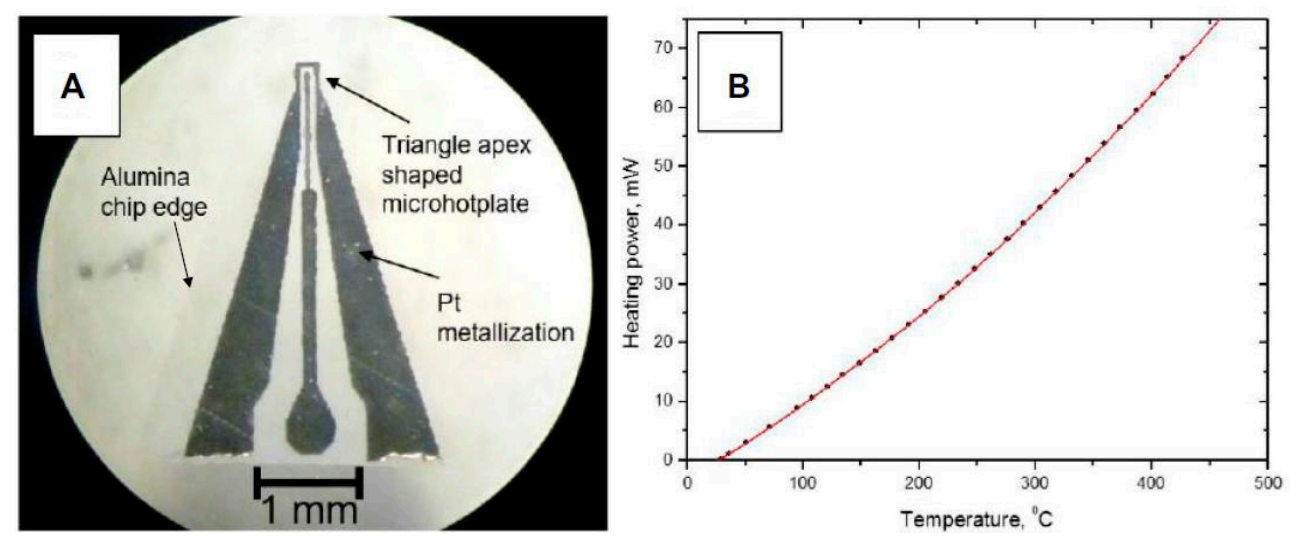

Figure 3. (A) Photo of the microheater fabricated by ink-jet printing of Pt based ink. The line width in the triangle apex part is of $50 \mu \mathrm{m}$, the microhotplate area of this microheater is of $\sim 300 \times 300 \mu \mathrm{m}$. (B) Power consumption of cantilever type microheater as a function of sensor working temperature.

The stability of the microheater was tested by applying constant voltage and by measurement of current through the microheater. The voltage was chosen to maintain constant temperature of the microheater equal to $450{ }^{\circ} \mathrm{C}$. The drift of the resistance is below $1 \%$ during 15 days of measurement. Typical operation mode of the sensor is pulsing heating with duty cycle of about $2.5 \%$ (approximately $250 \mathrm{~ms}$ of heating necessary to measure gas concentration with semiconductor gas sensor and $10 \mathrm{~s}$ interval between measurements). Therefore, under real exploitation conditions sensor remains working for more than one year.

\section{Conclusions}

We synthesized inks based on $\mathrm{Pt}, \mathrm{Au}$ and $\mathrm{Ag} 3-8 \mathrm{~nm}$ nanoparticles. The printability and applicability of Pt based ink was tested at the fabrication of microheaters of metal oxide gas sensors. The substrate of the sensor was $12 \mu \mathrm{m}$ thick anodic alumina. The microheater was prepared by Ink and AerosolJet technique, the microheater with $\sim 300 \times 300 \mu \mathrm{m}$ hot area consumes $\sim 70 \mathrm{~mW}$ at $450{ }^{\circ} \mathrm{C}$ and remains stable after at least 15 days of operation at this temperature corresponding to the operation in pulsing heating mode for $>1$ year.

Author Contributions: A.A.V. formulated the problem and fabricated ceramic MEMS sustrates for printing, V.P.K. prepared the samples of microheaters with ink jet printed Pt lines, S.V.T. synthesized Pt nanoparticles inks, D.Y.K. developed the instrumentation for ink preparation, S.P.G. is the author of original method of Pt nanoparticle synthesis, I.S.V. prepared the samples made using meniscus printing technique, I.E.J. tested the thermal properties of the microheaters, A.S.S. tested the printability of sensing materials onto microheater and tested their properties.

Acknowledgments: This work was supported by the Ministry of Education and Science of the Russian Federation (Grant No. 14.584.21.0020, unique identifier RFMEFI58416X0020). In the work, we used platinum ink given by Russian R\&D Company "AkKo Lab" (www.akkolab.ru) specialized in production of metal nanoinks for printed electronics.

Conflicts of Interest: The authors declare no conflict of interest.

\section{References}

1. Vasiliev, A.A.; Pisliakov, A.V.; Sokolov, A.V.; Samotaev, N.N.; Soloviev, S.A.; Oblov, K.; Guarnieri, V.; Lorenzelli, L.; Brunelli, J.; Maglione, A.; et al. Non-silicon MEMS platforms for gas sensors. Sens. Actuators B 2016, 224, 700-713.

2. Functional inks for inkjet-, aerosol- and screen-printing technologies - Printed electronics and sensor applications. Available online: https://www.ikts.fraunhofer.de/en/departments/energy_bio-medical_ technology/materials_and_components/HT_ElectrochemistryCatalysis/material_inks.html (accessed on 1 December 2018). 
3. Ramírez, J.L.; Annanouch, F.E.; Camara, M.; Lobet, E.L.; Briand, D. Single layer gold hotplate, printed on polyimide, with heater used as sensing current drain for metal-oxide gas sensor. Procedia Eng. 2015120 , 707-710.

4. Khan, S.; Nguyen, T.P.; Thiery, L.; Vairac, P.; Briand, D. Aerosol Jet Printing of Miniaturized, Low Power Flexible Micro-Hotplates. Proceedings 2017, 1, 316-320.

5. The MiCS-5524 is a compact MOS sensor. Available online: http://www.sgxsensortech.com/content/ uploads/2014/07/1084_Datasheet-MiCS-5524-rev-8.pdf (accessed on 1 December 2018).

(C) 2018 by the authors; Licensee MDPI, Basel, Switzerland. This article is an open access article distributed under the terms and conditions of the Creative Commons Attribution (CC BY) license (http://creativecommons.org/licenses/by/4.0/). 